\title{
Pancreas-specific Pten deficiency causes partial resistance to diabetes and elevated hepatic AKT signaling
}

\author{
Zan Tong ${ }^{1}$, Yan Fan ${ }^{2}$, Weiqi Zhang ${ }^{1}$, Jun Xu ${ }^{1}$, Jing Cheng ${ }^{3}$, Mingxiao Ding ${ }^{1,4}$, Hongkui Deng ${ }^{1,4}$ \\ ${ }^{1}$ Key Laboratory of Cell Proliferation and Differentiation of the Ministry of Education, College of Life Sciences, Peking University, \\ Beijing 100871, China; ${ }^{2}$ Key Laboratory of Bio-resource and Bio-environment, Ministry of Education, College of Life Science, \\ Sichuan University, Chengdu 610064, China; ${ }^{3}$ Beijing Laboratory Animal Research Center, Beijing 100012, China; ${ }^{4}$ Laboratory of \\ Chemical Genomics, Shenzhen Graduate School of Peking University, The University Town, Shenzhen 518055, China
}

PTEN, a negative regulator of the phosphatidylinositol-3-kinase/AKT pathway, is an important modulator of insulin signaling. To determine the metabolic function of pancreatic Pten, we generated pancreas-specific Pten knockout (PPKO) mice. PPKO mice had enlarged pancreas and elevated proliferation of acinar cells. They also exhibited hypoglycemia, hypoinsulinemia, and altered amino metabolism. Notably, PPKO mice showed delayed onset of streptozotocin (STZ)-induced diabetes and sex-biased resistance to high-fat-diet (HFD)-induced diabetes. To investigate the mechanism for the resistance to HFD-induced hyperglycemia in PPKO mice, we evaluated AKT phosphorylation in major insulin-responsive tissues: the liver, muscle, and fat. We found that Pten loss in the pancreas causes the elevation of AKT signaling in the liver. The phosphorylation of AKT and its downstream substrate GSK3 $\beta$ was increased in the liver of PPKO mice, while PTEN level was decreased without detectable excision of Pten allele in the liver of PPKO mice. Proteomics analysis revealed dramatically decreased level of 78-kDa glucose-regulated protein (GRP78) in the liver of PPKO mice, which may also contribute to the lower blood glucose level of PPKO mice fed with HFD. Together, our findings reveal a novel response in the liver to pancreatic defect in metabolic regulation, adding a new dimension to understanding diabetes resistance.

Keywords: Pten, pancreatic knockout, diabetes resistance, hepatic AKT signaling

Cell Research (2009) 19:710-719. doi: 10.1038/cr.2009.42; published online 14 April 2009

\section{Introduction}

Glucose homeostasis is tightly controlled by peripheral tissue sensitivity to insulin and insulin secretion. Impaired action and/or secretion of insulin cause diabetes [1-3]. PTEN (phosphatase and tensin homolog deleted on chromosome 10), originally discovered as a tumor suppressor $[4,5]$, was increasingly found to be impor-

Correspondence: Hongkui Deng

Tel: +86-10-6275-6474; Fax: +86-10-6275-6954

E-mail: hongkui_deng@pku.edu.cn

Abbreviations: STZ (streptozotocin); HFD (high-fat diet); X-Gal (5-bromo-4-chloro-3-indolyl $\beta$-D-galactoside); ALT (alanine aminotransferase); AST (aspartate aminotransferase); BUN (blood urea nitrogen); 2D-PAGE (two-dimensional polyacrylamide gel electrophoresis); ER (endoplasmic reticulum)

Received 12 November 2008; revised 7 December 2008; accepted 30 December 2008; published online 14 April 2009 tant in insulin signaling [6]. PTEN antagonizes the PI3K (phosphatidylinositol-3-kinase)-mediated signaling pathway by dephosphorylating PIP3 (phosphatidylinositol3,4,5-trisphosphate), an important secondary messenger responsive to insulin and insulin-like growth factor 1 (IGF-1) signaling [7-9]. Pten deficiency leads to accumulation of PIP3 and elevated activation of AKT [10]. AKT signaling plays an important role in glucose metabolism, protein synthesis, and adipogenesis [11-13].

Tissue-specific Pten knockout (PPKO) mouse models have shown that Pten has diverse biological roles depending on tissue types [14-25]. In major insulin target organs, principally the liver, muscle, and fat, Pten deficiency affects glucose metabolism and results in resistance to diabetes, suggesting that PTEN plays an important role in insulin action [19-22]. In the pancreas, PPKO led to age-dependent (arising in 2-3-month-old) ductal malignancy arising from the expansion of centroacinar cells [23]. $\beta$-cell-specific Pten deletion resulted 
in increased islet mass, enhanced $\beta$-cell survival, and altered glucose metabolism $[24,25]$. While both pancreatic endocrine and exocrine dysfunctions were reported to be correlated with malnutrition and diabetes [26, 27], the physiological role of pancreatic Pten in metabolism in vivo has not yet been fully elucidated.

To examine the role of pancreatic Pten in glucose homeostasis, we used a $P d x 1$ (pancreatic and duodenal homeobox gene 1) promoter-controlled Cre/loxP system to establish a complete pancreas-specific deletion of Pten. $P d x l$ is an essential transcription factor during pancreatic development $[28,29]$ and $P d x 1$-expressing cells can give rise to all three lineages of the pancreas (ducts, acini, and islets) [30]. In this study, we demonstrated that the pancreas-specific PPKO mice displayed increased acinar cell proliferation, and altered glucose and amino metabolism. Notably, we found that PPKO mice exhibited delayed onset of streptozotocin (STZ)-induced diabetes and sex-biased resistance to high-fat-diet (HFD)-induced diabetes. We further found elevated AKT signaling and decreased 78-kDa glucose-regulated protein (GRP78) level in the liver of PPKO mice. Both events may contribute to the resistance to HFD-induced hyperglycemia in PPKO mice.

\section{Results}

\section{Generation of pancreas-specific PPKO mice}

To generate pancreas-specific Pten deletion, we crossed Pten ${ }^{f l o x}$ ffox mice, in which the exons 4 and 5 of the Pten genes are flanked by the loxP sites, with $P d x 1-C r e$ transgenic mice. Pten ${ }^{f l o x /+} P d x 1-C r e^{-}$and $P t e n^{f l o x /+} P d x 1-$ $\mathrm{Cre}^{+}$mice were intercrossed to generate $P \operatorname{ten}^{f l o x}{ }^{f f o x} \mathrm{Pdx} 1$ $\mathrm{Cre}^{+}$(PPKO) mice, and we used the $\mathrm{Pten}^{+/+} \mathrm{Pdx} 1-\mathrm{Cr} \mathrm{e}^{+}$ mice as control. We confirmed that Pten was specifically deleted in the pancreas using various methods. First, we checked $P d x 1$-Cre mice using antibodies against PDX1 and CRE. Since PDX1 was expressed in the adult
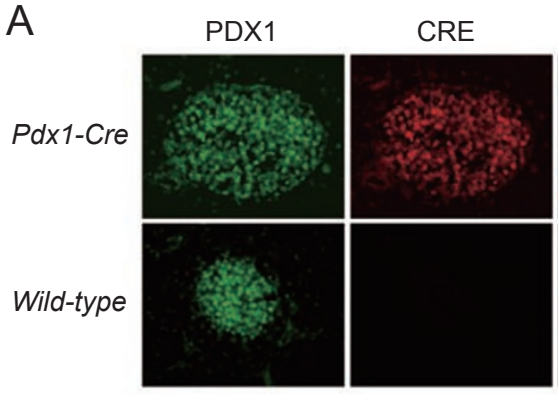

C

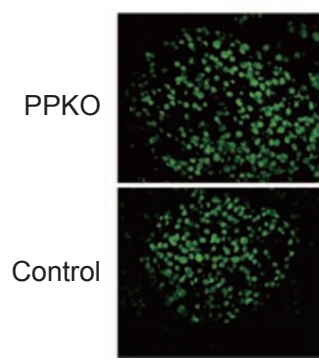

PTEN

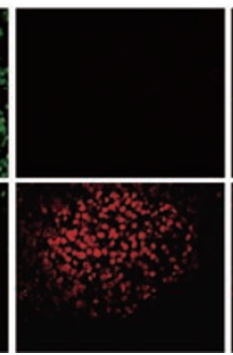

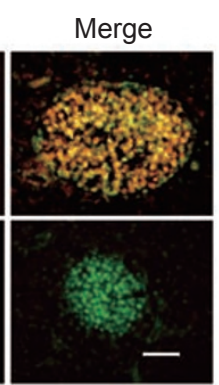

Merge

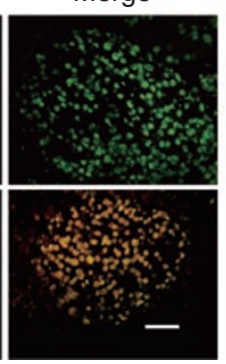

$B$

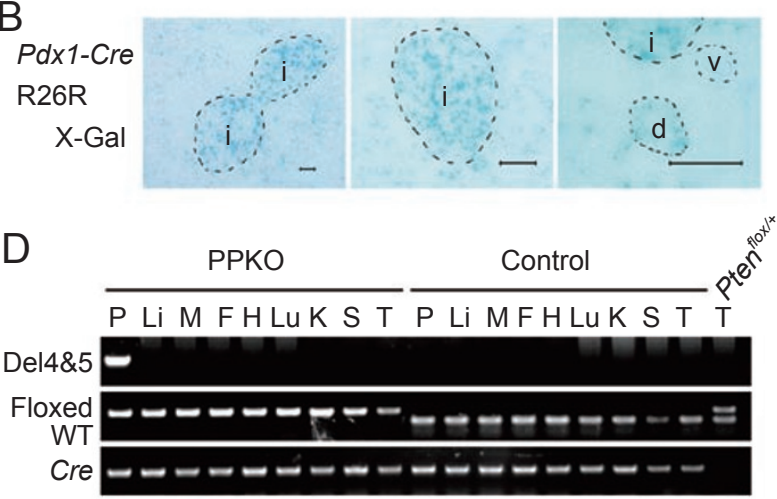

E

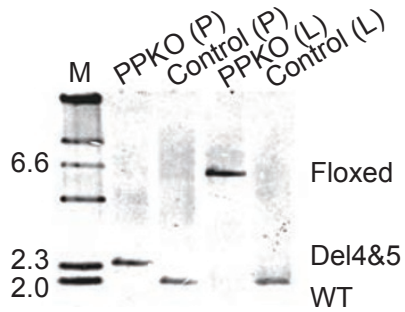

F

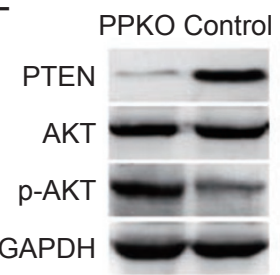

Figure 1 Generation of pancreas-specific Pten knockout mice. (A) Immunofluorescent detection of CRE in the pancreas of adult mice. CRE (red) colocalized with PDX1 (green) in Pdx1-Cre mice, while no CRE staining was seen in wild-type mice, bar $=50 \mu \mathrm{m}$. (B) X-Gal staining of pancreas sections of adult Pdx1-Cre; R26R (Rosa26 reporter) mice. Strong and dense $X-G a l$ staining was found in islets, while mosaic staining was seen in the exocrine. Staining was also found in duct cells but not in blood vessel, bar $=50 \mu \mathrm{m}$ (i, islet; d, duct; v, blood vessel). (C) Immunofluorescent detection of PTEN in the pancreas of adult mice. PTEN (red) costained with PDX1 (green) in control mice, while loss of PTEN was seen in PPKO mice, bar $=50$ $\mu \mathrm{m}$. (D) Genomic PCR analysis of Pten genotypes and Cre transgene in PPKO and control mice $(n=6)$. Pten deletion was found only in the pancreas (P) of PPKO mice, not in the liver (Li), skeleton muscle $(\mathrm{M})$, fat $(\mathrm{F})$, heart $(\mathrm{H})$, lung $(\mathrm{Lu})$, kidney $(\mathrm{K})$, spleen (S), or tail (T). (E) Southern blot analysis of genomic DNA from the liver (L) and pancreas (P) of PPKO and control mice $(n=3)$. Pten floxed allele: $6.0 \mathrm{~kb}$, Pten deleted allele: $2.3 \mathrm{~kb}$, Pten wild-type allele: $2.0 \mathrm{~kb}$. M, digoxigenin-labeled DNA marker ( $\lambda$-Hindlll digest). (F) Western blot analysis of protein lysates from the pancreas of PPKO and control mice $(n=3)$. Blots were probed with antibodies that recognized PTEN, AKT, p-AKT, and GAPDH. 
pancreatic islets, pancreatic sections from adult $P d x 1$ Cre mice showed co-staining of PDX1 and CRE in islets, while wild-type mice exhibited no CRE staining (Figure 1A). At the same time, liver sections from both PPKO and control mice exhibited no positive PDX1 and CRE staining (data not shown). Second, we assessed CRE activity by crossing ROSA26 reporter mice (B6; 129-Gt(ROSA)26Sor $\left.{ }^{t m / S h o} / \mathrm{J}\right)$ with the Pdxl-Cre mice. As reported previously [30], expression of lacZ was observed in all pancreatic lineages of adult mice (Figure 1B). Third, we showed PTEN co-localized with PDX1 in the islets of adult control mice, while it was lost in PPKO mice (Figure 1C). Fourth, we conducted PCR analysis of tissue-specific genomic DNA in adult mice. Pten deletion, as indicated by excision of the Pten locus (exons 4 and 5), was specifically found in the pancreas of PPKO mice (Figure 1D). Samples from control mice and the liver, skeleton muscle, fat, heart, lung, kidney, spleen, and tail of PPKO mice exhibited no excision of the Pten locus (Figure 1D). Fifth, we monitored Pten allele by Southern blot [15]. In PPKO mice, a 2.3-kb Pten deletion allele was found in the pancreas, while only the $6.0-\mathrm{kb}$ Pten floxed allele was detected in the liver (Figure 1E). In control mice, both the pancreas and liver exhibited the 2.0-kb Pten wild-type allele. Finally, we used western blot to determine PTEN protein levels in the pancreas. A residual amount of PTEN was observed in the pancreas of PPKO mice (Figure 1F), which might come from the slight contamination by non-pancreatic cells. We further examined AKT in the pancreas: the level of phosphoAKT (p-AKT) was increased in PPKO mice, while the level of total AKT protein was unaltered relative to control samples (Figure 1F).

\section{Enlarged pancreas and increased acinar cell prolifera- tion in PPKO mice}

We found that one-month-old PPKO mice exhibited normal body weight but a heavier pancreas (Supplementary information, Figure S1A). The weight ratio of pancreas to the total body increased markedly in PPKO mice compared with controls (Supplementary information, Figure S1A). As shown in Supplementary information, Figure S1B, we observed an increase in islet density and islet mass in PPKO mice. However, these results were not statistically significant. A markedly increased proliferation of acinar cells in PPKO mice was found using bromodeoxyuridine (BrdU) pulse labeling (Supplementary information, Figure S1C and S1D), while a trend toward increased proliferation of $\beta$-cells in PPKO mice was also noticed. Due to the low level of $\beta$-cell proliferation under static conditions, the difference in $\beta$-cell proliferation between PPKO and control mice was not statistically significant [31]. In addition, we found 6 out of 12 six-month-old PPKO mice showed a cystic presence of the pancreas, which exhibited extensive ductar complexes (Supplementary information, Figure S2).

\section{Altered glucose and amino metabolism in PPKO mice}

We examined the levels of various biochemical parameters in one-month-old PPKO and control mice (Table 1). Levels of fast blood glucose and fed serum insulin in PPKO mice were lower than those in the controls, while

Table 1 Biochemical analyses of PPKO and control mice

\begin{tabular}{llll}
\hline \multicolumn{1}{c}{ Index } & \multicolumn{1}{c}{ PPKO $(n)$} & Control $(n)$ & $P$ \\
\hline Blood glucose $(\mathrm{mM})$ & $8.7 \pm 0.8(10)$ & $6.5 \pm 0.9(12)$ & 0.053 \\
Fast blood glucose $(\mathrm{mM})$ & $5.3 \pm 0.8(16)$ & $0.52 \pm 0.14(10)$ & $0.028^{*}$ \\
Serum insulin $(\mathrm{ng} / \mathrm{ml})$ & $0.37 \pm 0.12(14)$ & $0.25 \pm 0.04(10)$ & $0.016^{*}$ \\
Fast serum insulin $(\mathrm{ng} / \mathrm{ml})$ & $0.21 \pm 0.04(10)$ & $103 \pm 11(8)$ & 0.052 \\
Pancreatic insulin $(\mathrm{ng} / \mathrm{mg})$ & $112 \pm 16(9)$ & $39.3(4)$ & 0.167 \\
Hepatic PEPCK activity $(\mathrm{nmol} / \mathrm{min} / \mathrm{mg})$ & $21.2(5)$ & $2849 \pm 106(9)$ & $0.0007^{* *}$ \\
Serum amylase $(\mathrm{U} / \mathrm{l})$ & $3499 \pm 143(7)$ & $290 \pm 31(8)$ & $0.006^{* *}$ \\
AST $(\mathrm{U} / \mathrm{l})$ & $278 \pm 28(8)$ & $129 \pm 8(8)$ & 0.877 \\
ALT $(\mathrm{U} / \mathrm{l})$ & $72 \pm 2(8)$ & $11.4 \pm 1.7(8)$ & $0.03 *$ \\
BUN $(\mathrm{mmol} / \mathrm{l})$ & $13.8 \pm 1.7(8)$ & $218 \pm 38(9)$ & $0.02^{*}$ \\
Blood ammonia $(\mu \mathrm{mol} / \mathrm{l})$ & $214 \pm 19(11)$ & $30.9 \pm 1.7(8)$ & 0.92 \\
Albumin $(\mathrm{g} / \mathrm{l})$ & $31.3 \pm 1.3(8)$ & $1.47 \pm 0.39(12)$ & 0.577 \\
Triglyceride $(\mathrm{mM})$ & $1.57 \pm 0.47(10)$ & $3.30 \pm 0.71(12)$ & 0.610 \\
Cholesterol $(\mathrm{mM})$ & $3.55 \pm 0.43(10)$ & 0.366 & \\
\hline
\end{tabular}

The number of one-month-old mice in each test was shown after each data in $(n)$. Data are presented as means \pm SEM. $* P<0.05 ; * * P<0.01$. 
the levels of fed blood glucose and fast serum insulin were maintained relatively normal in PPKO mice. The pancreatic insulin contents in one-month-old PPKO and controls were similar. The fast hepatic PEPCK activity was decreased in one-month-old PPKO mice compared with controls. Serum amylase, which is secreted by acinar cells, increased dramatically in the PPKO mice. We also examined amino metabolism-related indexes, and found blood urea nitrogen (BUN) increased markedly in PPKO mice, while blood ammonia did not change much; alanine aminotransferase (ALT) decreased in the PPKO mice, while aspartate aminotransferase (AST) remained normal. Serum albumin, triglyceride (TG), and cholesterol (CHO) in PPKO mice remained similar to controls (Table 1). In order to evaluate the insulin sensitivity of the PPKO and control mice, we performed glucose tolerance test (GTT) and insulin tolerance test (ITT). In GTT, one-month-old PPKO mice demonstrated significantly lower levels of peak blood glucose at $15 \mathrm{~min}$ (by $27 \%$, $P<0.05)$ and the blood glucose levels returned to the same level at 120 min in both PPKO and control groups
(Supplementary information, Figure S3A). The increased glucose clearance in GTT also occurred in four-monthold groups of PPKO mice (Supplementary information, Figure S3B), but was not as observable as in one-monthold mice groups. These results suggest that the PPKO mice had an increased ability to dispose of the injected glucose. PPKO mice, both one-month and four-months old, exhibited nearly normal insulin sensitivity in ITT (Supplementary information, Figure S3C and S3D).

\section{Delayed onset of STZ-induced diabetes in PPKO mice}

To determine the pathophysiological significance of the Pten in the pancreas, we treated PPKO and control mice with low doses of STZ. As shown in Figure 2A, control mice showed elevated blood glucose after STZ injection and maintained a hyperglycemic state $(>12.7$ $\mathrm{mM}$ ) from days 5 to 24 before being killed (31.7 \pm 2 $\mathrm{mM})$. In contrast, PPKO mice treated with STZ exhibited dramatically lower glucose levels during the first 3 days post-injection, similar to vehicle-injected control mice. From day 4 to day 6, PPKO mice injected with STZ
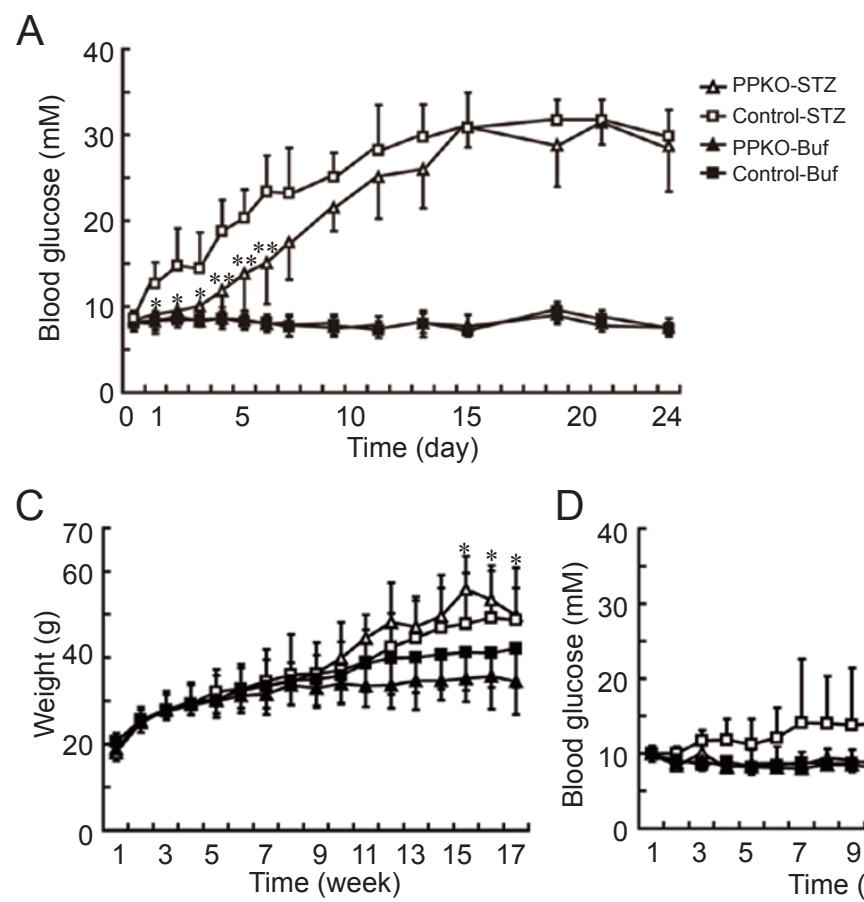

$B$
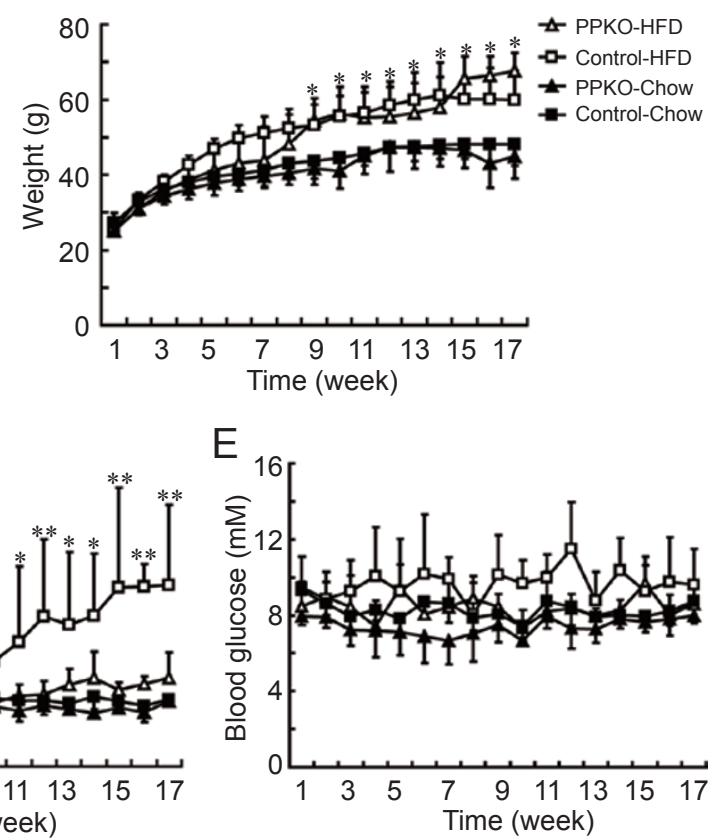

Figure 2 Partial resistance to STZ- and HFD-induced hyperglycemia in PPKO mice. (A) Multiple low-dose STZ was injected into 1-month-old PPKO and control mice; citrate buffer (Buf) was injected as control. Each group $n=9$ (5 male and 4 female), a total of four groups. Blood glucose levels were measured before the first injection (day 0 ) and after the final injection for 24 days (day 1 to day 24). (B-E) PPKO mice and controls, 1-month old, were fed with HFD for 17 weeks, or fed normal chow as control. The body weight and blood glucose, measured once a week, were plotted against weeks on the diet. Male mice: each group $n=9$, a total of four groups. Female mice: each group $n=8$, a total of four groups. Both male (B) and female (C) mice had steady increase of body weight fed with HFD. (D) Blood glucose was increased in male control mice fed with HFD, while it was maintained in male PPKO mice fed with HFD. (E) Normal blood glucose in female PPKO and control mice fed with HFD. $* P<0.05 ; * * P<0.01$. 
exhibited rapidly increased glucose levels. From day 15 on, PPKO mice showed hyperglycemia similar to control mice receiving STZ injection $(30.9 \pm 3 \mathrm{mM})$. Therefore, PPKO mice showed delayed onset of low-dose STZinduced diabetes.

\section{Resistance to HFD-induced hyperglycemia in PPKO mice}

To further investigate whether pancreatic Pten deficiency prevented type 2 diabetes, we fed PPKO and control mice with HFD. A sexual bias of HFD-induced diabetes was observed. During the 17 weeks on HFD, both PPKO and control mice exhibited increased body weight (Figure 2B and 2C). Male control mice exhibited increased blood glucose levels and began to show hyperglycemia after 7 weeks on HFD (Figure 2D). At the same time, male PPKO mice fed with HFD maintained normal blood glucose levels similar to those of mice fed with regular diet (Figure 2D). From 11 weeks onward, male control mice fed with HFD had dramatically higher blood glucose levels than those of PPKO mice (Figure 2D). Thus, male PPKO mice were resistant to HFDinduced hyperglycemia. We found female mice did not develop hyperglycemia during HFD test (Figure 2E), despite being overtly obese (Figure 2C).

\section{Increased AKT phosphorylation in the liver of PPKO mice}

As the resistance to HFD-induced hyperglycemia could be caused by changes in peripheral insulin signaling, we analyzed levels of phosphorylated AKT in the major insulin-responsive organs (liver, fat, and muscle) by western blot. In the fat and muscle, p-AKT levels in PPKO mice were indistinguishable from those in controls; however, in the liver, p-AKT level was markedly increased in PPKO mice compared with controls (Figure $3 \mathrm{~A})$. We further demonstrated that the level of phosphoGSK3 $\beta$ (p-GSK3 $\beta$ ), a major downstream target of AKT, was increased in the liver of PPKO mice, while the total protein levels of AKT and GSK3 $\beta$ remained unchanged (Figure 3B).

We found that the level of PTEN in the liver of PPKO mice was approximately half of that in control and Pten-

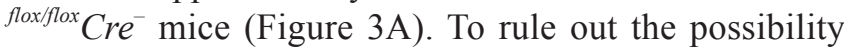
that the Pten alleles may be excised in the liver of PPKO mice and this non-specific Pten deletion is responsible for the increase of p-AKT levels, we used genome PCR and Southern blot to monitor the Pten alleles (Figure 1D and 1E). These analyses confirmed that Pten deletion occurred only in the pancreas of PPKO mice and was undetectable in the liver of PPKO mice.

In order to find factors affecting Pten expression in the liver of PPKO mice, we used quantitative RT-PCR to check the mRNA levels of p53, c-Jun, Egrl, and RelA,

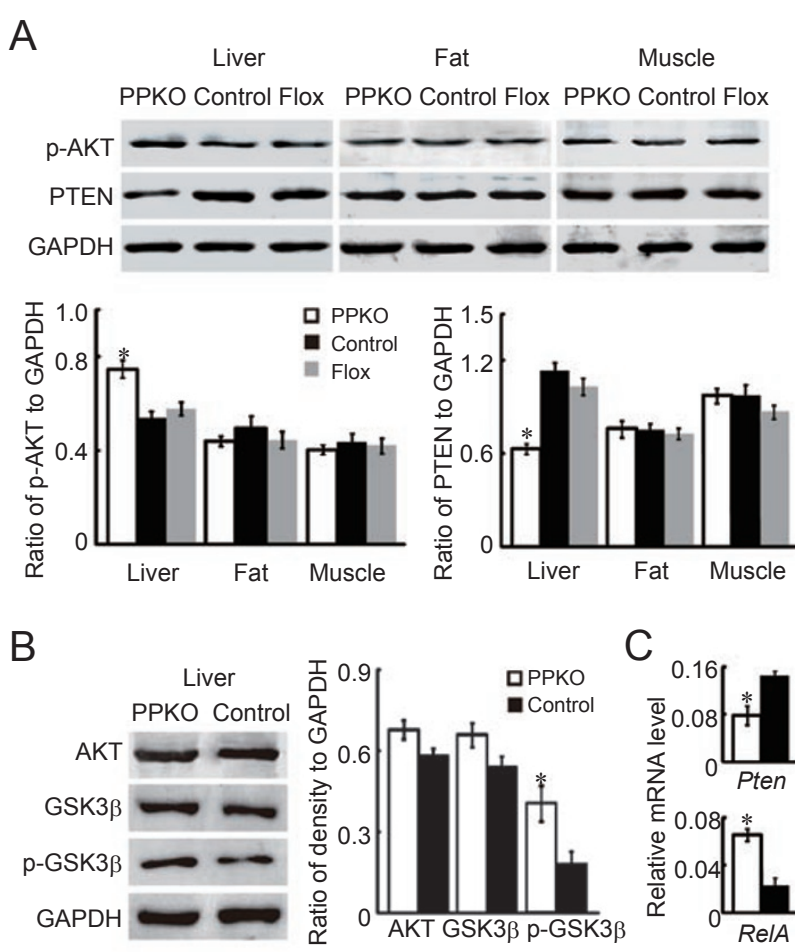

Figure 3 Increased AKT signaling in the liver of PPKO mice. (A) Western blot analysis of protein lysates isolated from the liver, fat, and muscle of 1-month-old PPKO, control, and Pten${ }^{\text {floxflox }} \mathrm{Cre}^{-}$(Flox) mice $(n=3)$. Blots were probed with anti-pAKT, PTEN, and GAPDH (as loading controls) antibodies. (B) Western blot analysis of protein lysates isolated from the liver of 1-month-old PPKO and control mice $(n=3)$. Blots were probed with anti-AKT, GSK3 $\beta$, p-GSK3 $\beta$, and GAPDH antibodies. (C) Quantitative RT-PCR analysis of the mRNA level of Pten and RelA in the liver of PPKO and control mice $(n=3) .{ }^{*} P<0.05$.

which encode proteins reported directly regulating Pten expression [32-35]. We found the mRNA level of RelA was markedly increased in the liver of PPKO mice, while the level of Pten was decreased (Figure 3C). At the same time, the mRNA levels of p53, c-Jun, and Egrl showed no difference between PPKO and control mice (data not shown).

\section{Proteomics analysis of the liver}

To identify additional changes in the liver of PPKO mice, we conducted a proteomics analysis comparing the hepatic proteins from six PPKO and control mice (Figure 4A). We found six major differentially expressed proteins (Figure 4B), and the differences were further confirmed by western blot analysis (Figure 4C). These six identified proteins could be divided into two groups according to their reported functions. The first group includes the GRP78, nuclear transcription factor Y subunit gamma (NFYC), and eukaryotic translation initia- 
A

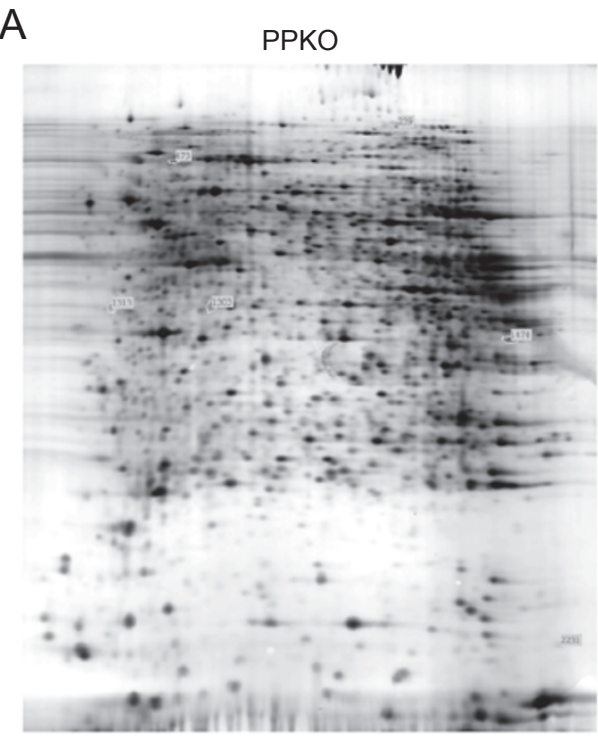

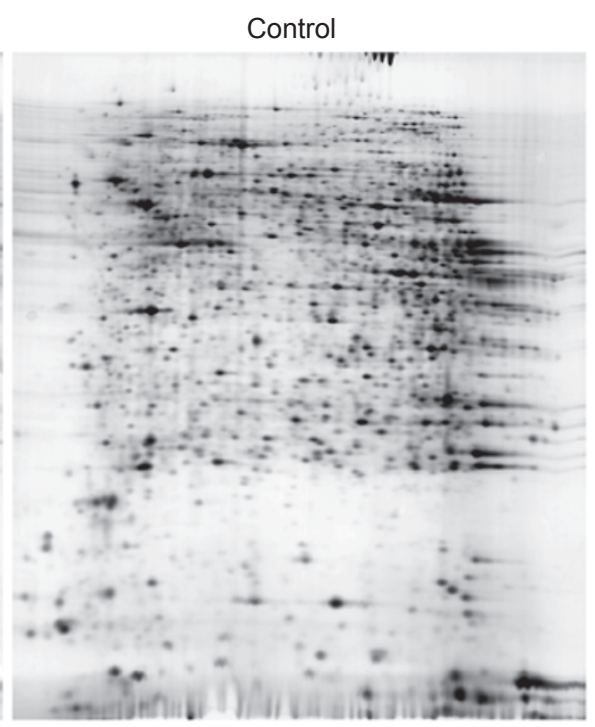

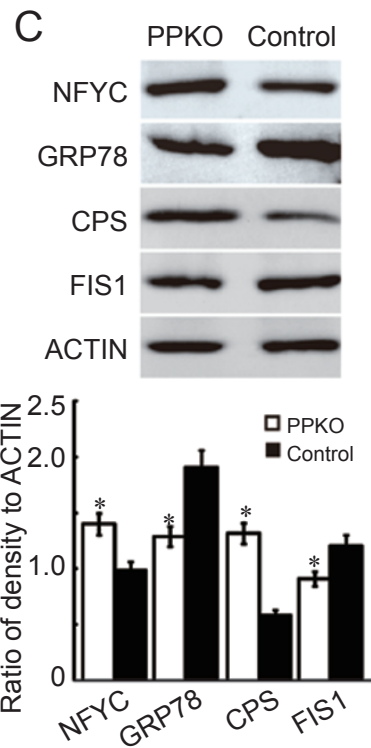

B

\begin{tabular}{rlr}
\hline Spot No. & \multicolumn{1}{c}{ Protein } & $\begin{array}{c}\text { Fold } \\
\text { PPKO vs Control }\end{array}$ \\
\hline 1313 & Nuclear transcription factor Y subunit gamma (NFYC) & 1.64 \\
1303 & Eukaryotic translation initiation factor 3 subunit 2 (elF3 $\beta$ ) & -1.51 \\
473 & 78 kDa glucose regulated protein precursor (GRP78) & -3.56 \\
239 & Carbamoyl-phosphate synthase [ammonia] mitochondrial precursor (CPS) & 3.16 \\
1474 & Dehydrogenase/reductase SDR family member 1 (DHRS1) & 1.94 \\
2231 & Mitochondrial fission 1 protein (FIS1) & -1.60 \\
\hline
\end{tabular}

Figure 4 Liver proteomics analysis of PPKO and control mice. (A) Silver-staining images of hepatic proteins. Proteins from liver homogenates of 1 -month-old mice $(n=6)$ were mixed together for 2-DE PAGE analysis. The hepatic proteins were separated by isoelectric focusing ( $\mathrm{pH} 3$ to 10 , horizontal axis) and 12.5\% SDS-PAGE (vertical axis). The differential protein spots were showed in the image of PPKO mice. (B) Identities of the six differentially expressed proteins and fold changes revealed by proteomics. $P \leq 0.05$. (C) Four differentially expressed proteins were confirmed by western blot of protein lysates from the liver of 1-month-old PPKO and control mice $(n=3)$. Blots were probed with antibodies that recognized NFYC, GRP78, CPS, FIS1, and ACTIN. *P<0.05.

tion factor 3 subunit 2 (eIF3 $\beta$ ). GRP78, an endoplasmic reticulum (ER) chaperone [36], showed decreased levels in the liver of PPKO mice compared with controls. NFYC is a subunit of NFY, which is a CCAATbinding transcription factor and has been reported to be required for Grp78 expression through interaction with CCAAT motif in the Grp78 promoter [37]. NFYC had an increased level in the liver of PPKO mice. eIF3 $\beta$, an important factor recruited to ER-bound ribosome for translation initiation [38], showed decreased levels in the liver of PPKO mice. The second group consists of three mitochondrial proteins: carbamoyl phosphate synthase (CPS), dehydrogenase/reductase SDR (short-chain dehy- drogenases/reductases) family member 1 (DHRS1), and mitochondrial fission 1 protein (FIS1). CPS and DHRS1, both important in the urea cycle [39], showed increased levels in the liver of PPKO mice, while FIS1, functional in mitochondrial fission and apoptosis [40], displayed a modestly decreased level in PPKO mice.

\section{Discussion}

In this study, we demonstrated that PPKO mice had hypoglycemia, hypoinsulinemia, and partial resistance to induced diabetes. Our results also revealed an unsuspected increase of hepatic AKT signaling and decrease 
of hepatic GRP78 level in response to pancreatic Pten deficiency. These intriguing changes in the liver may be important for the resistance to HFD-induced hyperglycemia in PPKO mice.

In the present study, we found pancreatic Pten deletion results in elevated hepatic AKT signaling. Both AKT and GSK3 $\beta$, a major downstream effector of AKT, showed increased phosphorylation in the liver of PPKO mice (Figure 3A). Pten expression was decreased (Figure 3A) and the possible deletion of Pten in the liver of PPKO mice was excluded by genomic PCR and Southern blot analysis (Figure 1D and 1E). A previous study reported that adipose-specific PPKO also led to increased PI3K/ AKT signaling in the liver [21]. Moreover, hepatic PI3K activity was reported to be responsive to either adiposespecific Glut4 deletion [41] or muscle-specific uncoupling protein 3 (Ucp3) overexpression [42]. Our findings, together with these previous observations, implied that hepatic PI3K/AKT signaling was sensitive to metabolic changes in other insulin function-related tissues: fat, muscle, and pancreas.

What is the major signal leading to decreased hepatic PTEN and increased hepatic AKT signaling in PPKO mice? The increased mRNA level of RelA (Figure 3C) may associate with the down-regulation of Pten in the liver of PPKO mice, since RelA was reported to bind to the promoter of Pten and suppress Pten expression [34]. On the other hand, PPKO mice had low serum insulin (Table 1). Chronic hypoinsulinemia has been reported to affect insulin actions in the liver [43], which possibly contributes to the increased hepatic AKT signaling in PPKO mice. Further studies are required to identify molecules that mediate the induction of decreased hepatic PTEN and increased hepatic AKT signaling in PPKO mice.

Our results reveal that PPKO mice were resistant to HFD-induced hyperglycemia (Figure 2D). One possible mechanism for the resistance could be increased hepatic AKT signaling. Previous studies reported that hepatic overexpression of AKT induced lower blood glucose [44] and inhibition of PTEN expression in liver and muscle could reverse hyperglycemia in diabetic mice [6]. Specific GSK-3 inhibitor treatment was reported to lower hyperglycemia in diabetic rats by increasing liver glycogen synthesis [45]. In addition, we found decreased level of GRP78, an ER stress-responsive protein [36], in the liver of PPKO mice (Figure 4C). ER stress-responsive proteins were reported to show increased expression in the liver of diabetic mice [46] and reducing ER stress in the liver could restore glucose homeostasis in diabetic mice [47]. Together, both elevated AKT signaling and decreased GRP78 level in the liver of PPKO mice may contribute to the resistance to HFD-induced hyperglycemia.

On the other hand, our results demonstrated that PPKO mice had altered amino metabolism. Dramatic increased level of CPS (Figure 4C), a critical enzyme in the urea cycle, in the liver was consistent with increased blood urea levels in PPKO mice (Table 1). This increased BUN could be partly due to increased serum amylase in PPKO mice, since increased serum amylase was reported to be associated with increased nitrogen loss [48].

Altogether, we have found novel responses of the liver to pancreatic PPKO: elevated AKT signaling, decreased GRP78, and increased CPS. Further investigation of the molecular basis for the responses in PPKO mice may bring new insight for understanding the cross-talk between the pancreas and the liver.

\section{Materials and Methods}

\begin{abstract}
Animals
Pten ${ }^{f l o x f l o x}$ mice with exons 4 and 5 of Pten flanked by loxP sites, kindly offered by Dr Tak Wah Mak [15], were mated to Pdx1-Cre transgenic mice (C57/B6), in which Cre was expressed under the control of $P d x 1$ promoter. The $P d x 1$-Cre plasmid was a kind gift

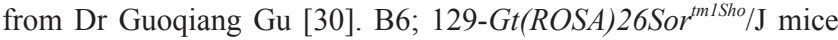
were kindly offered by the Model Animal Research Center of Nanjing University. All mice were housed in pathogen-free facilities on a 12-h light-dark cycle. All animal-handling procedures were in compliance with the standards established by the Animal Care and Ethics Committee of Peking University.
\end{abstract}

\section{PCR analysis}

Genomic DNA from tissues was isolated and amplified by PCR as described. Primers used for the floxed Pten (335 bp) and wildtype Pten (228 bp): 5'-CTC CTC TAC TCC ATT CTT CCC-3' and 5'-ACT CCC ACC AAT GAA CAA AC-3'; primers for deleted Pten (849 bp): 5'-GTC ACC AGG ATG CTT CTG AC-3' and 5'ACT ATT GAA CAG AAT CAA CCC-3'; primers for Cre $(480$ bp): 5'-GCC TGC ATT ACC GGT CGA TGC-3' and 5'-CAG GGT GTT ATA AGC AAT CCC-3'.

\section{Quantitative RT-PCR}

RNA was extracted from liver using RNeasy Micro kit (Qiagen). Equivalent amounts of cDNA samples were derived from $50 \mathrm{ng}$ of DNase-digested total RNA using Omniscript RT Kit (Qiagen). Quantitative PCR analysis was performed on ABI PRISM 7300 Sequence Detection System using the SYBR Green PCR Master Mix (TOYOBO). The threshold cycle values were determined using the AUTOANALYSE features of Sequence Detection System software. Quantitative PCR was performed in duplicate for each sample, and three independent experiments were carried out. The relative RNA level of each gene was normalized against $\beta$-actin. Primers used for Pten: 5'-AAA GGG ACG GAC TGG TGT AAT G -3' and 5'-AGC AGT GCC ACG GGT CTG TAA T-3' (200 bp); p53: 5'-CAT CAC CTC ACT GCA TGG AC-3' and 5'-AAA AGA TGA CAG GGG CCA TG-3' (172 bp); c-Jun: 5'-CCA GAA GAT GGT GTG GTG TT-3' and 5'-AGA TTC AAA GTT GGA AGG AG-3' (221 bp); Egrl: 5'-AAA AGC ACA GGA GGG A-3' and 
5'-GGC AGG GAT GGT AAG T-3' (133 bp); RelA: 5'-CTG TGC CTA CCC GAA AC-3' and 5'-GCA GAG GAC GGT GCC AA-3' (159 bp); $\beta$-actin: $5^{\prime}$ - AGG TCA TCA CTA TTG GCA ACG A-3' and 5'- CAC TTC ATG ATG GAA TTG AAT GTA GTT-3' (117 bp).

\section{Western blot analysis}

Tissue samples were homogenized using PRO-PREP Protein Extraction Solution (iNtRON Biotechnology, Korea). Seventy micrograms of protein was loaded on SDS-10\% polyacrylamide gel electrophoresis (PAGE) gel. Blots were probed with antibodies that recognized PTEN, AKT, p-AKT (Ser473), GSK3 $\beta$ (27C10), and phospho-GSK3 $\beta$ (Ser9) from Cell Signaling Technology. Antibody to CPS was from Santa Cruz Biotechnology, to FIS1 was from MBL, to GRP78 was from Calbiochem, and to NFYC was from Protein Tech Group, Inc. The same membranes were probed with anti-GAPDH or anti-ACTIN from Santa Cruz Biotechnology as loading controls. Signal densities of western blot were analyzed using Image-Pro Plus software.

\section{Southern blot analysis}

Genomic DNA was extracted from the pancreas and the liver. Ten micrograms of DNA was digested with HindIII and separated by electrophoresis in $1 \%$ agarose gel. DNA was transferred to $\mathrm{Ny}-$ lon membrane and Pten alleles were detected using 604 bp probe. Primers for the probe: 5'-GCT CCA TTG GGC TGT AGT TT$3^{\prime}$ and 5'-CAT CCT GGT GAC TCC AAC TA-3'. The probe was labeled with digoxigenin and detected using a DIG Nucleic Acid Detection Kit (Roche).

\section{Histochemistry}

$\mathrm{LacZ}$ expression was visualized on $5-\mu \mathrm{m}$-thick pancreatic cryosections by 5-bromo-4-chloro-3-indolyl $\beta$-D-galactoside (X-Gal) (Promega) staining overnight at $37{ }^{\circ} \mathrm{C}$. Immunofluorescence staining was performed using $5-\mu \mathrm{m}$-thick pancreatic cryosections. Primary antibodies (rabbit anti-INSULIN, rabbit anti-glucagon, and mouse anti-BrdU) were obtained from Santa Cruz Biotechnology. Rabbit anti-AMYLASE was from Sigma, rabbit anti-cytokeratin (pan) was from Zymed and rabbit anti-CRE was from Novagen. Antibodies recognized PDX1 were kindly offered by Dr C Wright. The secondary antibodies were TRITC-conjugated donkey antirabbit IgG, FITC-conjugated donkey anti-goat IgG, and FITCconjugated donkey anti-mouse IgG (Jackson Immunoresearch). Nuclei were detected by DAPI (Sigma) staining.

\section{Determination of islet cell mass and cell proliferation}

Pancreatic slides $120 \mu \mathrm{m}$ apart from 1-month-old PPKO or control mice were counted. Stained pancreatic sections $(5 \mu \mathrm{m}$ thick) were analyzed using Image-Pro Plus software. Islet area was determined from at least 1000 islets (more than five insulin positive cells). Islet mass was calculated by multiplying pancreas weight by the percentage of islet area in pancreatic section area. Islet density was determined by dividing the number of islet by pancreatic section area. Cell proliferation was evaluated using BrdU pulse labeling. One-month-old PPKO and control mice were i.p. injected with $\mathrm{BrdU}(100 \mathrm{mg} / \mathrm{kg}$ of body weight) and killed $1 \mathrm{~h}$ later. $\beta$-Cell proliferation was measured as the percentage of BrdU and INSULIN double-positive cells of the total number of INSULIN-positive cells. Acinar cell proliferation was expressed as the ratio of BrdU and AMYLASE double-positive cells to the pancreatic section area.

\section{Biochemical analysis}

Blood samples from animals were collected between 9 a.m. and 10 a.m.. Glucose levels were assessed using a glucometer (GLUCOTrend 2, Roche). Serum insulin concentrations were assayed using Rat/Mouse Insulin ELISA Kit (LINCO). One-month-old mice were fasted overnight before following biochemical analysis. Pancreatic insulin content was determined after extracted pancreas by cold acid-ethanol and normalized for pancreas weight. Serum amylase was measured using VITR $\phi$ S system chemistry 950 . Serum levels of albumin, TG, $\mathrm{CHO}$, and BUN were determined by colorimetric enzymatic assays following the manufacturers' protocols (Biosino Biotechnology Company Ltd, China and Beckman Coulter). Blood ammonia was measured using DRI-CHEM100.

\section{Hepatic PEPCK assay}

PEPCK activity was determined by the decarboxylation assay described by Jomain-Baum and Schramm [49]. Liver from overnight fasted mice was homogenized in the buffer $(10 \mathrm{mmol} / \mathrm{l}$ HEPES and $0.25 \mathrm{~mol} / 1$ sucrose, $\mathrm{pH}$ 7.4). The homogenate was centrifuged at $12000 \mathrm{rpm}$ for $15 \mathrm{~min}$ at $4{ }^{\circ} \mathrm{C}$ and the supernatant was used to assay for PEPCK activity. The 20 - $\mu$ l-homogenate-added $1 \mathrm{ml}$ action buffer $\left(50 \mathrm{mmol} / \mathrm{l}\right.$ Tris $\mathrm{pH} 8.0,0.75 \mathrm{mmol} / 1 \mathrm{MnCl}_{2}, 1$ $\mathrm{mmol} / 1 \mathrm{NAD}+, 6 \mathrm{U}$ malate dehydrogenase, $1 \mathrm{mmol} / 1 \mathrm{GTP})$ was incubated at $37^{\circ} \mathrm{C}$ for $5 \mathrm{~min}$. The reaction was initiated by adding $10 \mathrm{mmol} / \mathrm{l}$ malate, and the formation of NADH was monitored by absorbance at $340 \mathrm{~nm}$ for $5 \mathrm{~min}$. PEPCK activities were expressed as nmol/min NADH formation per microgram cytosol protein. The protein concentration $(\mathrm{g} / \mathrm{ml})$ in cytosol preparations was determined using BCA protein assay kit (PIERCE).

\section{GTTS and ITTS}

For GTTs, mice were fasted overnight for 14-16 h followed by i.p. injection of $20 \%$ glucose ( $2 \mathrm{~g} / \mathrm{kg}$ of body weight). Blood glucose levels were determined at $0,15,30,60,90$, and 120 min after injection. For ITTs, mice were fasted for 4-5 h, followed by i.p. injection of recombinant human insulin (Humulin R, Lilly, China, $1 \mathrm{U} / \mathrm{kg}$ of body weight). Blood glucose levels were determined at 0 , $15,30,45$, and 60 min after injection.

\section{STZ experiment}

One-month-old PPKO mice and controls after $4 \mathrm{~h}$ fast were i.p. injected with STZ (50 mg/kg of body weight) in citrate buffer (Sigma) or an equivalent volume of citrate buffer daily for consecutive 5 days. Blood glucose levels were measured before the first injection and after the final injection between 9 a.m. and 10 a.m..

\section{HFD experiment}

One-month old PPKO mice and controls were fed HFD (35.5\% fat) (\#F3282, Bio-Serve, Frenchtown, NJ) or normal diet as control for 17 weeks. Body weight and blood glucose levels were measured weekly between 9 a.m. and 10 a.m..

\section{Proteomics study}

Livers from six PPKO mice and six controls, 1-month old, were stored at $-80{ }^{\circ} \mathrm{C}$ before being sent to the Research Center for Proteome Analysis (RCPA) (http://www.proteomics.ac.cn/). Protein samples were separated by two-dimensional (2D)-PAGE 
and visualized using silver staining. The stained gels were scanned using a Bio-Rad GS710 scanner and analyzed using Image Master. Differential expressed protein spots were cut from gels and subjected to digestion and ESI Mass Spectrometry. By searching the IPI mouse protein library, the differentially expressed proteins were identified.

\section{Statistical analysis}

All values are expressed as mean \pm standard error of the mean. Differences were analyzed using the two-tailed unpaired Student's $t$-test and $P \leq 0.05$ considered statistically significant.

\section{Acknowledgments}

This research was supported by grants from the Ministry of Education (705001), National Basic Research Program of China (973 Program 2009CB941200), National Natural Science Foundation of China (30830061 and 30421004), and a 111 project to H Deng. We thank Dr Tak Wah Mak (University of Alberta, Canada) for kindly providing the Pten ${ }^{f l o x f f o x}$ mice, Dr Guoqiang Gu (Vanderbilt University, USA) for kindly providing the plasmid of Pdx1-Cre, and Dr C Wright (Vanderbilt University, USA) for the PDX1 antibody. We thank the Model Animal Research Center of Nanjing University for B6; 129-Gt(ROSA) 26 Sor $^{\mathrm{tm} 1 \mathrm{Sho}} / \mathrm{J}$ mice and the Research Center for Proteome Analysis for proteomics analysis. We thank Dr Matt Stremlau, Dr Hui Zhang, Jun Cai, Han Qin, Jian Li, Yan Shi, Haisheng Zhou, and Fei Ye for their critical reading of the manuscript. We also thank Wei Jiang, Yushan Guo, Jie Yang, Chengyan Wang, Hui Zhang, and other colleagues in our laboratory for providing technical assistance and advice during the experiments.

\section{References}

1 Martin BC, Warram JH, Krolewski AS, et al. Role of glucose and insulin resistance in development of type 2 diabetes mellitus: results of a 25-year follow-up study. Lancet 1992; 340:925-929.

2 Kahn CR. Banting Lecture. Insulin action, diabetogenes, and the cause of type II diabetes. Diabetes 1994; 43:1066-1084.

3 Baudry A, Leroux L, Jackerott M, Joshi RL. Genetic manipulation of insulin signaling, action and secretion in mice. Insights into glucose homeostasis and pathogenesis of type 2 diabetes. EMBO Rep 2002; 3:323-328.

4 Li J, Yen C, Liaw D, et al. PTEN, a putative protein tyrosine phosphatase gene mutated in human brain, breast, and prostate cancer. Science 1997; 275:1943-1947.

5 Steck PA, Pershouse MA, Jasser SA, et al. Identification of a candidate tumour suppressor gene, MMAC1, at chromosome $10 \mathrm{q} 23.3$ that is mutated in multiple advanced cancers. Nat Genet 1997; 15:356-362.

6 Butler M, McKay RA, Popoff IJ, et al. Specific inhibition of PTEN expression reverses hyperglycemia in diabetic mice. Diabetes 2002; 51:1028-1034.

7 Maehama T, Dixon JE. The tumor suppressor, PTEN/MMAC1, dephosphorylates the lipid second messenger, phosphatidylinositol 3,4,5-trisphosphate. J Biol Chem 1998; 273:1337513378.

8 Stambolic V, Suzuki A, de la Pompa JL, et al. Negative regulation of PKB/Akt-dependent cell survival by the tumor suppres- sor PTEN. Cell 1998; 95:29-39.

9 Saltiel AR, Kahn CR. Insulin signalling and the regulation of glucose and lipid metabolism. Nature 2001; 414:799-806.

10 Stocker H, Andjelkovic M, Oldham S, et al. Living with lethal PIP3 levels: viability of flies lacking PTEN restored by a $\mathrm{PH}$ domain mutation in Akt/PKB. Science 2002; 295:2088-2091.

11 Ueki K, Yamamoto-Honda R, Kaburagi Y, et al. Potential role of protein kinase $\mathrm{B}$ in insulin-induced glucose transport, glycogen synthesis, and protein synthesis. J Biol Chem 1998; 273:5315-5322.

12 Gagnon A, Chen CS, Sorisky A. Activation of protein kinase $\mathrm{B}$ and induction of adipogenesis by insulin in 3T3-L1 preadipocytes: contribution of phosphoinositide-3,4,5-trisphosphate versus phosphoinositide-3,4-bisphosphate. Diabetes 1999; 48:691-698.

13 Cho H, Mu J, Kim JK, et al. Insulin resistance and a diabetes mellitus-like syndrome in mice lacking the protein kinase Akt2 (PKB beta). Science 2001; 292:1728-1731.

14 Backman SA, Stambolic V, Suzuki A, et al. Deletion of Pten in mouse brain causes seizures, ataxia and defects in soma size resembling Lhermitte-Duclos disease. Nat Genet 2001; 29:396-403.

15 Suzuki A, Yamaguchi MT, Ohteki T, et al. T cell-specific loss of Pten leads to defects in central and peripheral tolerance. Immunity 2001; 14:523-534.

16 Suzuki A, Itami S, Ohishi M, et al. Keratinocyte-specific Pten deficiency results in epidermal hyperplasia, accelerated hair follicle morphogenesis and tumor formation. Cancer Res 2003; 63:674-681.

17 Suzuki A, Kaisho T, Ohishi M, et al. Critical roles of Pten in B cell homeostasis and immunoglobulin class switch recombination. J Exp Med 2003; 197:657-667.

18 Backman SA, Ghazarian D, So K, et al. Early onset of neoplasia in the prostate and skin of mice with tissue-specific deletion of Pten. Proc Natl Acad Sci USA 2004; 101:1725-1730.

19 Horie Y, Suzuki A, Kataoka E, et al. Hepatocyte-specific Pten deficiency results in steatohepatitis and hepatocellular carcinomas. J Clin Invest 2004; 113:1774-1783.

20 Stiles B, Wang Y, Stahl A, et al. Liver-specific deletion of negative regulator Pten results in fatty liver and insulin hypersensitivity [corrected]. Proc Natl Acad Sci USA 2004; 101:20822087.

21 Kurlawalla-Martinez C, Stiles B, Wang Y, et al. Insulin hypersensitivity and resistance to streptozotocin-induced diabetes in mice lacking PTEN in adipose tissue. Mol Cell Biol 2005; 25:2498-2510.

22 Wijesekara N, Konrad D, Eweida M, et al. Muscle-specific Pten deletion protects against insulin resistance and diabetes. Mol Cell Biol 2005; 25:1135-1145.

23 Stanger BZ, Stiles B, Lauwers GY, et al. Pten constrains centroacinar cell expansion and malignant transformation in the pancreas. Cancer Cell 2005; 8:185-195.

24 Nguyen KT, Tajmir P, Lin CH, et al. Essential role of Pten in body size determination and pancreatic beta-cell homeostasis in vivo. Mol Cell Biol 2006; 26:4511-4518.

25 Stiles BL, Kuralwalla-Martinez C, Guo W, et al. Selective deletion of Pten in pancreatic beta cells leads to increased islet mass and resistance to STZ-induced diabetes. Mol Cell Biol 2006; 26:2772-2781. 
26 Lombardo F, De Luca F, Rosano M, et al. Natural history of glucose tolerance, beta-cell function and peripheral insulin sensitivity in cystic fibrosis patients with fasting euglycemia. Eur J Endocrinol 2003; 149:53-59.

27 Cavalot F, Bonomo K, Perna P, et al. Pancreatic elastase-1 in stools, a marker of exocrine pancreas function, correlates with both residual beta-cell secretion and metabolic control in type 1 diabetic subjects. Diabetes Care 2004; 27:2052-2054.

28 Jonsson J, Carlsson L, Edlund T, Edlund H. Insulin-promoterfactor 1 is required for pancreas development in mice. Nature 1994; 371:606-609.

29 Offield MF, Jetton TL, Labosky PA, et al. PDX-1 is required for pancreatic outgrowth and differentiation of the rostral duodenum. Development 1996; 122:983-995.

$30 \mathrm{Gu}$ G, Dubauskaite J, Melton DA. Direct evidence for the pancreatic lineage: $\mathrm{NGN} 3+$ cells are islet progenitors and are distinct from duct progenitors. Development 2002; 129:2447-2457.

31 Bonner-Weir S, Weir GC. New sources of pancreatic betacells. Nat Biotechnol 2005; 23:857-861.

32 Virolle T, Adamson ED, Baron V, et al. The Egr-1 transcription factor directly activates PTEN during irradiation-induced signalling. Nat Cell Biol 2001; 3:1124-1128.

33 Stambolic V, MacPherson D, Sas D, et al. Regulation of PTEN transcription by p53. Mol Cell 2001; 8:317-325.

34 Vasudevan KM, Gurumurthy S, Rangnekar VM. Suppression of PTEN expression by NF-kappa B prevents apoptosis. Mol Cell Biol 2004; 24:1007-1021.

35 Hettinger K, Vikhanskaya F, Poh MK, et al. c-Jun promotes cellular survival by suppression of PTEN. Cell Death Differ 2007; 14:218-229.

36 Munro S, Pelham HR. An Hsp70-like protein in the ER: identity with the $78 \mathrm{kd}$ glucose-regulated protein and immunoglobulin heavy chain binding protein. Cell 1986; 46:291-300.

37 Roy B, Li WW, Lee AS. Calcium-sensitive transcriptional activation of the proximal CCAAT regulatory element of the grp78/BiP promoter by the human nuclear factor CBF/NF-Y. $J$ Biol Chem 1996; 271:28995-29002.

38 Valasek L, Nielsen KH, Hinnebusch AG. Direct eIF2-eIF3 contact in the multifactor complex is important for translation initiation in vivo. EMBO J 2002; 21:5886-5898.

39 Hinsdale ME, Hamm DA, Wood PA. Effects of short-chain acyl-CoA dehydrogenase deficiency on development expression of metabolic enzyme genes in the mouse. Biochem $\mathrm{Mol}$ Med 1996; 57:106-115.

40 Lee YJ, Jeong SY, Karbowski M, Smith CL, Youle RJ. Roles of the mammalian mitochondrial fission and fusion mediators Fis1, Drp1, and Opa1 in apoptosis. Mol Biol Cell 2004; 15:5001-5011.

41 Abel ED, Peroni O, Kim JK, et al. Adipose-selective targeting of the GLUT4 gene impairs insulin action in muscle and liver. Nature 2001; 409:729-733.

42 Choi CS, Fillmore JJ, Kim JK, et al. Overexpression of uncoupling protein 3 in skeletal muscle protects against fat-induced insulin resistance. J Clin Invest 2007; 117:1995-2003.

43 Lopez S, Burlet H, Desbuquois B. Mechanisms of up-regulation of the liver insulin receptor in chronically hypoinsulinemic rats: assessment of receptor endocytosis. Mol Cell Endocrinol 1991; 82:159-164.

44 Ono H, Shimano H, Katagiri H, et al. Hepatic Akt activation induces marked hypoglycemia, hepatomegaly, and hypertriglyceridemia with sterol regulatory element binding protein involvement. Diabetes 2003; 52:2905-2913.

45 Cline GW, Johnson K, Regittnig W, et al. Effects of a novel glycogen synthase kinase-3 inhibitor on insulin-stimulated glucose metabolism in Zucker diabetic fatty $(\mathrm{fa} / \mathrm{fa})$ rats. Diabetes 2002; 51:2903-2910

46 Ozcan U, Cao Q, Yilmaz E, et al. Endoplasmic reticulum stress links obesity, insulin action, and type 2 diabetes. Science 2004; 306:457-461.

47 Ozcan U, Yilmaz E, Ozcan L, et al. Chemical chaperones reduce ER stress and restore glucose homeostasis in a mouse model of type 2 diabetes. Science 2006; 313:1137-1140.

48 Havala T, Shronts E, Cerra F. Nutritional support in acute pancreatitis. Gastroenterol Clin N Am 1989; 18:525-542.

49 Jomain-Baum M, Schramm VL. Kinetic mechanism of phosphoenolpyruvate carboxykinase (GTP) from rat liver cytosol. Product inhibition, isotope exchange at equilibrium, and partial reactions. J Biol Chem 1978; 253:3648-3659.

(Supplementary information is linked to the online version of the paper on the Cell Research website.) 